\title{
Designing for Behavior Change - 6 Dimensions of Social Comparison Features
}

\author{
Vasiliki Mylonopoulou \\ University of Oulu \\ vasiliki.mylo@oulu.fi
}

\author{
Karin Väyrynen \\ University of Oulu \\ karin.vayrynen@oulu.fi
}

\author{
Minna Isomursu \\ IT University of Copenhagen \\ miis@itu.dk
}

\begin{abstract}
Social comparison as an aspect of social influence has an effect on health behavior, and technology can be used to support desired behavior change. However, no concrete guidelines exist on how to design social comparison features. This paper examines how designers have actually designed social comparison in IT artifacts supporting individuals in a behavior change process. We apply qualitative evidence synthesis review method and analyze twelve studies reporting experiences of designing social comparison features. As a result, we present six design dimensions for social comparison features emerging from the literature, and several alternative design options for each dimension. The dimensions can be used as a guide for designers and as a repository for researchers to design and evaluate social comparison features for technologies targeting behavior change in different contexts.
\end{abstract}

\section{Introduction}

Behavior change is a process happening in stages, and risk of relapse into the old behavior is usually high [21]. Different types of technological applications have been developed to support this challenging process $[10,17,19,31]$. Social influence features are commonly used in these technologies, because social influence has an effect on people's behavior, for example, increase the time people bike instead of using other transportation means [31], or motivate them to go jogging in the morning [23]. Designers have to be mindful when engineering technologies using social influence [23] and its aspects [22]. One aspect of social influence is social comparison [6, 18, 22]. Even though research exists on how technology can support the behavior change through social influence - using theories and models from behavior change, social influence and persuasive technology $[4,8,10,13,15,16,19,24]-$ it is open and abstract regarding the social comparison as a social influence aspect. Even though patterns have been studied for other social influence aspects, e.g., cooperation, competition, and social learning [20], for social comparison this type of research is missing. In this paper, we address this gap and focus on the features of technological applications that support behavior change utilizing the aspect of social comparison: the comparison between the data of individuals [6]. We look into the social comparison features design that present the relation between the data of an individual and that of others to better understand the design solutions which were inspired by the applied theories. This will give an idea about the existing design alternatives, and it will give the possibility to further examine these alternatives. We attempt to answer the following research question:

How can social comparison features be designed for technology that targets behavior change?

\section{Social comparison in social influence}

Social comparison is many times connected with competition against others. However, social comparison theory has little to do with competition in the social psychology context. It was introduced by Festinger [6] in the 1950s, describing the people's tendency to compare with others similar to them for self-evaluation regarding skills and opinions. Later, downwards [28] and upwards [5] social comparison were distinguished to express the comparison with someone inferior or superior to oneself, respectively.

Social comparison has various scientific applications in healthcare [3]. Women with breast cancer, e.g., compared themselves with breast cancer patients worse than them on coping with the disease, even if that patients were fabricated (i.e. imaginary situations the patient never saw or experienced) [30]. Another example is Gibbons et al.'s [11] research where they showed that smokers who affiliate with people who had difficulties to quit smoking had a bigger relapse rate than those who affiliate themselves with people who had an easier time quitting.

The design of technologies targeting behavior change is influenced by social comparison and other 
social studies $[13,24,26]$ as well as health behavior change models [19]. These technologies targeting behavior change are also described as persuasive technologies [7]. Social influence [1] has a big part in persuasive technologies [7] and it has been identified to affect health behavior such as an increase in physical activity, e.g., biking [31], jogging [23], and walking [19]. However, many technologies have interpreted social influence in various ways in their design [4, 10, 19, 25]. It is recently that social influence has been studied in depth in the context of technology and systems in a way to illustrate its different aspects [22] and applications, such as social comparison [18]. No specific guidelines on the application of social comparison in persuasive technology exist. However, designers - who have perceived and apply social influence in different ways - have implemented social comparison features.

This paper aims to identify and categorize the common characteristics of social comparison features' design by analyzing the design of these features in published articles. These categories will reveal the different dimensions of social comparison which can be used to design social comparison features and also draw a clearer image on what designs exist, to allow evaluate their efficiency in different contexts at a later stage.

\section{Methodology}

To be able to answer the research question, our review focuses on articles which report development and validation of IT artifacts (technological interventions such as mobile applications, computer applications, embedded technology etc.) that support behavior change, and visualize the comparison between the data of an individual and that of others.

Qualitative evidence synthesis review [12] is the research method selected to answer the research question of this paper as its goal is interpretive and allows to understand a phenomenon and generate generalizations by finding patterns in the literature. Comprehensive search strategies as well as more selective approaches to identify relevant qualitative studies are seen acceptable in this method [12].

Data collection was conducted through Google Scholar in order to find as many relevant articles as possible, regardless of the specialization field, citations amount, or publication place/period- in three search rounds. First, we used all combinations of terms that could be related to technology targeting to change health or other behavior through social influence, i.e.: visualization, visualisation, behavior change, behaviour change, behavioral change, behavioural change, persuasion, health, social influence, and persuasive technology. At the end of the first search round, more field-specific terminology was found through the keywords and the background section of the retrieved articles: infovis, social capital, social visualization, social comparison, habitual behavior, and habitual behaviour. All keywords were discussed with an information specialist at the University of Oulu for evaluation and validation. Second we used all combinations of the first round's keywords and the field-specific keywords that resulted from the first round. Third, backwards snowball technique [29] was used to find more papers through the reference lists of the papers found. The inclusion criteria were:

1. The user had to be involved in the evaluation of the artifact. Artifacts not tested in such way were discarded, because it was impossible to see their effect on user's behavior.

2. The IT artifact presents the relation between the individual's and others' data. Artifacts showing only comparisons between two individuals or groups were discarded to make the study focused.

3. The paper demonstrates influence of the IT artifact on the users' behavior.

First, the articles were examined by the title and abstract. Articles obviously unrelated to the subject of behavior change and technology were excluded. Next, we read the whole article or until the point at which one of the above criteria was unsatisfactory.

The first criterion was evaluated by reading the abstract and methodology of the reviewed paper to identify user studies. If no user involvement was mention, the article was excluded. For the second criterion, we analyzed the artifact's characteristics, which were described in a separate section in each article and were usually accompanied with pictures of the artifact. Here, we evaluated whether the artifact included any social features that fulfilled our second inclusion criterion. The third criterion was analyzed based on the participants' choices and intentions referred in the evaluation, result, or discussion section of the article. The search resulted in 12 articles fulfilling all criteria, two of which described the same artifact. Three artifacts targeted health behavior, five ecological behavior, one both health and ecological behavior, and two other behaviors. Due to limited amount of artifacts targeting health behavior change we examine all eleven artifacts.

After the relevant articles were collected, an affinity diagram was created [2] to visualize the categories found in the thematic analysis and a thematic analysis [14] conducted to find patterns in the design of social comparison features. Table 1 summarizes basic information about these artifacts: a code (A1-A11) used in the rest of the text to refer the 
Table 1. Reviewed articles and IT artifacts

\begin{tabular}{|c|c|c|}
\hline \# & Paper Title & Description \\
\hline $\mathrm{A} 1$ & $\begin{array}{l}\text { Fish'n'Steps: Encouraging Physical } \\
\text { Activity with an Interactive Computer } \\
\text { Game [19] }\end{array}$ & $\begin{array}{l}\text { Technology: (mainly) personal computer, pedometer. Aim: motivate users to be } \\
\text { physically active. Design: the users were represented by fishes in a fish tank. The fish's } \\
\text { mood was related to user's physical activity. If the fish's mood became too bad, the } \\
\text { environment in the tank got bad. Theoretical group: persuasive technology and behavior } \\
\text { change. Behavior change: Health }\end{array}$ \\
\hline$\overline{\mathrm{A} 2}$ & $\begin{array}{l}\text { Social Visualization Encouraging } \\
\text { Participation in Online Communities } \\
\text { [24] }\end{array}$ & $\begin{array}{l}\text { Technology: personal computer. Aim: motivate students to be active in the online } \\
\text { community. Design: The students were represented by stars, the star's size represented } \\
\text { the users' activity, and the color represented the most common activity of the user } \\
\text { (upload/download a file). Theoretical group: social influence. Behavior change: Other }\end{array}$ \\
\hline $\mathrm{A} 3$ & $\begin{array}{l}\text { Persuasiveness of a Mobile Lifestyle } \\
\text { Coaching Application Using Social } \\
\text { Facilitation [10] }\end{array}$ & $\begin{array}{l}\text { Technology: personal computer or mobile phone. Aim: motivate users to eat fruits and } \\
\text { be physically active. Design: The users were grouped in teams. The data of the team } \\
\text { members were presented next to each other. A smiley represented the daily, personal goal } \\
\text { completion. Two progress bars - which consisted of small squares (slots) - represented } \\
\text { the vegetables portions, and the exercise sessions respectively. Theoretical group: } \\
\text { persuasive technology and behavior change. Behavior change: Health }\end{array}$ \\
\hline A4 & $\begin{array}{l}\text { Design and Evaluation of a Social } \\
\text { Visualization aimed at Encouraging } \\
\text { Sustainable Behavior [13] }\end{array}$ & $\begin{array}{l}\text { Technology: personal computers. Aim: motivate the users to adopt environment friendly } \\
\text { activities. Design: The users were represented by puzzle pieces, the more environment } \\
\text { friendly activities a user made, the clearer and lighter their piece became. Theoretical } \\
\text { group: social influence. Behavior change: ecological. }\end{array}$ \\
\hline A5 & $\begin{array}{l}\text { inAir: Sharing Indoor Air Quality } \\
\text { Measurements and Visualizations [25] }\end{array}$ & $\begin{array}{l}\text { Technology: gadget/domestic display (paced in private houses), personal computer was } \\
\text { used to visualize the individual long term data. Aim: air quality awareness. Design: A } \\
\text { line graph showed the air quality of each user's place the last three seconds. A bar graph } \\
\text { showed the current air quality of each place. Theoretical group: persuasive technology. } \\
\text { Behavior change: Health: }\end{array}$ \\
\hline A6 & $\begin{array}{l}\text { UpStream: Motivating Water } \\
\text { Conservation with Low-Cost Water } \\
\text { Flow Sensing and Persuasive Displays } \\
{[17]}\end{array}$ & $\begin{array}{l}\text { Technology: gadget placed on water sources (faucets and showers). Aim: water } \\
\text { preservation. Design: Multiple versions of the gadget were studied. 1. Light variation } \\
\text { based on water consumption: green light symbolized that the current consumption is } \\
\text { under the average, yellow that it is around the average, red that it is over the average. 2. } \\
\text { Progress bar divided in sections representing the accumulative water consumption (the } \\
\text { more water consumed the more sections turned on). 3. Numerical gallon visualization of } \\
\text { the current (dynamic) and the average (static) water consumption. Theoretical group: } \\
\text { based on past technological applications. Behavior change: ecological }\end{array}$ \\
\hline A7 & $\begin{array}{l}\text { The Design and Evaluation of Prototype } \\
\text { Eco-Feedback Displays for Fixture- } \\
\text { Level Water Usage Data [8] }\end{array}$ & $\begin{array}{l}\text { Technology: domestic display. Aim: water preservation. Design: the residence water } \\
\text { consumption was visualized in various ways (not all presented). 1. Graphs visualized the } \\
\text { inhabitants' water consumption, in relation to each inhabitant's overall consumption (line } \\
\text { graphs), and in relation to different activities i.e., shower (bar graphs). 2. A digital } \\
\text { aquarium, the state of which represented the overall water consumption. Theoretical } \\
\text { group: based on past technological applications. Behavior change: ecological }\end{array}$ \\
\hline A8 & $\begin{array}{l}\text { GreenSense: Developing Persuasive } \\
\text { Service Technology by Integrating } \\
\text { Mobile Devices and Social Interaction } \\
\text { for Sustainable and Healthy Behavior [4] }\end{array}$ & $\begin{array}{l}\text { Technology: (mainly) mobile phones, public display. Aim: motivate the use of } \\
\text { transportation means to commute (environmental and health related). Design: The public } \\
\text { display through a colorful bar graph presented: the users' "green credit", gained by the } \\
\text { users' actions (bar's length); and what transportation mean was chosen (bar's colors). } \\
\text { Theoretical group: social influence. Behavior change: Health and ecological }\end{array}$ \\
\hline A9 & $\begin{array}{l}\text { Reveal-it!: The Impact of a Social } \\
\text { Visualization Projection on Public } \\
\text { Awareness and Discourse [26] }\end{array}$ & $\begin{array}{l}\text { Technology: public displays, tablet. Aim: raise energy consumption awareness. Design: } \\
\text { The users entered the money corresponding to their electricity consumption (tablet). On } \\
\text { display: rectangles represent the users, its' size showed the electricity consumption, its' } \\
\text { colors showed the neighborhood. The neighborhood's maximum energy consumption } \\
\text { was highlighted. The neighborhood's and overall average were shown by lines crossing } \\
\text { the rectangles. Theoretical group: social influence. Behavior change: ecological }\end{array}$ \\
\hline \multirow[t]{2}{*}{ A10 } & $\begin{array}{l}\text { BizWatts: A modular socio-technical } \\
\text { energy management system for } \\
\text { empowering commercial building } \\
\text { occupants to conserve energy [15] }\end{array}$ & \multirow[t]{2}{*}{$\begin{array}{l}\text { Technology: personal computers. Aim: energy preservation in commercial buildings. } \\
\text { Design: Diagrammatic visualization of the users' energy consumption. Theoretical } \\
\text { group: based on past technological applications. Behavior change: ecological. }\end{array}$} \\
\hline & $\begin{array}{l}\text { Effects of real-time eco-feedback and } \\
\text { organizational network dynamics on } \\
\text { energy efficient behavior in commercial } \\
\text { buildings [16] }\end{array}$ & \\
\hline A11 & $\begin{array}{l}\text { MyPosition: Sparking Civic Discourse } \\
\text { by a Public Interactive Poll Visualization } \\
\text { [27] }\end{array}$ & $\begin{array}{l}\text { Technology: public displays. Aim: raise awareness on people's opinion on different } \\
\text { topics and trigger conversations. Design: The display presented a question and four } \\
\text { answers. The users chose an answer (by standing in front of it and raise their hand). A } \\
\text { picture of them voting was taken, placed in a square, and the square was placed under the } \\
\text { chosen answer together with the other users' squares with the same opinion. Theoretical } \\
\text { group: based on past technological applications. Behavior change: other }\end{array}$ \\
\hline
\end{tabular}


corresponding artifact, the title of the article reviewed, and a description of the artifact presented in the article. Due to limited amount of artifacts targeting health behavior change, we examined all artifacts. However, the result of the article can be applied in health behavior change support systems as well as other behavior change support systems.

\section{Results and discussion}

The analysis resulted in six design dimensions for social comparison features, each of which includes several design alternatives. We present and discuss each dimension through examples from the reviewed literature (see Table 1). Due to page limitation only representative examples are presented in detail.

\subsection{Strategies to influence behavior}

4.1.1. Awareness increase. Artifacts that influenced or aimed to influence people's awareness and social communication used public displays (A8, A9, A11). A8 (aimed to influence how people commute) had both mobile and public display interface. Social comparison was presented on the public display with little success because of the visualizations' complexity and stagnation (bar graph). A11 (aimed to raise awareness/trigger conversations by visualizing people's opinions) triggered conversations between people who were in the area without interacting actively with the display. In A9 (aim to raise energy consumption awareness) the people playfully pressured their peers to share their electricity consumption data and then reflected and comment on the results presented on public display.

In the transtheoretical model, people in the first behavior change stages need to become motivated to take an action towards a new behavior in the near future [21]. If a designer targets the first stage of behavior change and awareness raise, a well-designed public display may be a solution.

4.1.2. Immediate feedback. Four artifacts (A2, A5, A6, A7) gave immediate feedback to the users about their actions. In general, getting the right information on the right time may be crucial for decision making - as it has been discussed in The Design of EcoFeedback Technology [9].

An example of immediate feedback application is A6, which informed the users how much water they consumed, in relation to the average water consumption (through light variation) at the same time and place they used the water source. The users became aware of the water usage the moment they used the water source. This led to subconscious behavior change on water consumption.

Immediate feedback does not mean use of ubiquitous/embodied technology but technology which is bounded with the actions connected to the behavior. A2 was a PC application which targeted to motivate students to use a file-sharing community by connecting the activity of the user to a personal staravatar. The application gave feedback about the users' actions at the time they used it, by changing the star's attributes.

To conclude, immediate feedback can be used to make the users aware of their actions and progress while they are doing the activity connected to the behavior they want to change. Immediate feedback can be also used as a reminder/warning when they diverge from the desirable behavior.

4.1.3. Tracking. Several of the IT artifacts analyzed in this review were applications for tracking people's behavior (A1, A3, A4, A8, A10), resembling an activity diary. The users were required to input the data of the activity they tried to change in a cellphone or computer. The application stored and visualized their progress and how their progress related to others. A representative example is A3 where the user's eating and exercising data is presented in two corresponding bars. The target for each user was to take seven actions per day, e.g., eat three portions of vegetables and conduct four times 10 minute exercise in a day. The users were required to input manually the portions of vegetables they ate and the blocks of exercise they did. After that they were able to see their progress and see how they were doing in relation to their team members.

The aim of tracking is to inform people in a visual way about their actions, how these actions are related to their target and the progress of others. A common characteristic of these artifacts is that people get informed only when they access the application, so they stay uninformed about their progress if they do not take the action to enter the application.

\subsection{Types of the comparison}

4.2.1. Compare with all users of the artifact. Most of the IT artifacts (A2, A3, A5, A7, A8, A10) visualized the progress of all or many participants in the same screen, either by placing users' avatars in the same digital environment (A2: stars in the sky), or by visualizing each user as a different line/bar in a graph (e.g. A10). Thus, the users could compare concurrently their individual progress with that of others and consequently with the overall progress of the presented users. A10 - in addition to visualize 
multiple users' data - showed the user's individual goal in relation to the rest of the group. The user could, e.g., see a line-graph that relates his/hers progress and target to the progress of eight users.

By visualizing each user, they could relate with each other, understand their place in the group, and see how many people do better or worse than them.

4.2.2. Compare with aggregated users' data. Some IT artifacts (A6, A11, A7) showed the relation between an individual and an aggregated value extracted from the data of all users such us average, minimum, maximum, or sum. For example, the users could see their progress in comparison with the average progress of all users (A6: water consumption comparison through light variation); they could see if their behavior or attitude was in the minority, majority, or somewhere in between (A11: users placed as squares under the choice they made); and they could see how their progress could influence the result of the whole group (A6, A7: the water consumption is related to a digital aquarium's state). A6 also visualized how the individual water consumption adding on the overall.

Having aggregated values, e.g., average, minimum, and maximum, helps to keep the users' data anonymized, gives an overall idea of the user in the group, or the user's contribution to the group result (A6) and eliminates any competitive feelings between the individuals because they cannot compare with someone but with a faceless anonymized value.

4.2.3. Combination. Some IT artifacts used both comparison types in the same visualization or group of visualizations (A1, A3, A4, A9). In A3, the users were placed into teams and they could compare with the team's members and overall progress. A1, and A4 showed the relation between users and how one user's actions influenced the overall group progress. In A9, the users could compare with: all the users, their group's average, the overall average, the overall and the group's minimum/maximum.

The combination of comparison types gives a better understanding on users' place in the group, facilitates individual comparisons, and makes visible the distance from the best or worst performance.

\subsection{Dimensions of the comparison}

4.3.1. One-dimensional comparison. Onedimensional visualizations show only the relation between the current state of a user and the others (A1, A4, A6, A11). For example, in A1 the avatar-fishes showed the relation between the users' current physical activity states, and in A6 the light variations represented the user's current water consumption in relation to the overall average water consumption.

With one-dimensional comparisons, users are unable to see their progress as they can only see their current state. However, one-dimensional comparisons may have a direct effect on users' behavior when combined with immediate feedback e.g., A6: users changed their behavior based the comparison between their current water consumption and the average water consumption.

4.3.2. Two-dimensional comparison. Twodimensional comparisons show the relation between the data of an individual and that of others using two comparable variables (A2, A5). For example in A2, the users could compare their star-avatars based on their size and color. The size showed the amount of files someone downloaded/uploaded, while the color showed which action (upload/download) was more common. In A5 the users could compare their room's air quality current state and its state in time.

This comparison can show the relation between the users at the current moment but also in time. It can show the comparison between two variables e.g., frequency and type of an activity.

4.3.3. Multi-dimensional comparison. Some artifacts visualized more than two dimensions (A3, A7-A10). For example, in A3 the users could compare several data sets (eating habits, exercise habits, and target completion) with other users. In A9 the user could compare their electricity consumption data with: other users, neighborhoods, the neighborhood's average, and the overall average.

The different dimensions of a comparison may give the users a better understanding on their behavior change as they can compare with different variables e.g., their progress, current state, aggregated values (average, minimum, maximum etc.), etc. However, the more dimensions a comparison has, the more complicated the visualization becomes. Thus, multidimensional comparisons should be designed cautiously to avoid confusing visualizations.

\subsection{Attitudes promoted}

4.4.1. Competitive attitude. Some artifacts (A1-A3, A5, A7-10) promoted competitive attitude, explicitly by rewarding users who performed better (A1, A3, A7), or implicitly by presenting the results in a way that the best performers came on the top of a list or diagram (A2, A5, A8-A10). 
The competitive attitude may demotivate some users who dislike to compete [8]. However, that may be avoided if it is combined with cooperative attitude (e.g. A1, A3, A7, A10).

4.4.2. Cooperative attitude. Artifacts promoting cooperative attitude use social comparison to show how the behavior of an individual contributes to shared goals (A1, A3, A4, A7, A6, A10). Some artifacts (A3, A10) divided users in teams so they could cooperate to compete with another team. In A3 for example, the users could see how they influenced their team's progress and its relation to other teams. Other artifacts (A1, A4, A6, A7) showed the individual's influence on the common virtual environment. For example, in A1 every user was represented by a fish avatar placed in a fish tank together with other fishes (users). The state of the avatar was related to its owner's physical activity. If the owner was inactive, the avatar got sad. If that continued, the fish tank's environment got unpleasant for all the users. Another example is A6 that visualized how the user's water consumption contributed to the accumulative water consumption.

The cooperative attitude can be promoted by grouping users in teams, visualizing the users' accountability, visualizing the users' effect on the common environment, visualizing a situation and the importance of each user's impact on a common goal.

\subsection{Types of social comparison visualizations}

4.5.1. Abstract visualizations. This visualization use shapes or light variations, without forming any specific "character", i.e., fish, star, face etc.

A4 (and A11) used squares to represent users. In A4 for example, each square was a piece in a puzzle. If the users performed poorly on taking eco-friendly actions, their piece in the puzzle got darker and affected the overall puzzle image.

Variation in the color of light is another way to show the relation between the data of an individual and that of many. One A6's iteration visualized the user's water consumption in relation to the average, based on light's colors: green light, showed under the average water consumption; yellow around the average; and red above the average. The paper's authors [17] noticed that the light variation visualization is more memorable than a numerical visualization. However, in specific context (public toilets) the artifact was perceived as playful, people experimented with their water consumption to see the artifact's functionalities and this resulted in the undesired behavior (increased water consumption).
To conclude, abstract visualizations in public spaces should be vivid, in order to draw people's attention; and clear on their purpose, in order to motivate people to use the IT artifact and to demotivate them to play with it.

4.5.2. Avatar and avatar like visualizations. This category includes anthropomorphized and nonanthropomorphized avatars (A1-A3). In particular, digital entities connected to individual's progress.

In A1 the avatar-fishes' facial expressions (happy, sad, and angry) were connected to their owner's physical activity. The avatars engaged emotionally the users: users showed their avatars to other people to show their progress; but when they had poor performance they avoided looking at the sad fishes.

In one of A2's iterations the metaphorical staravatar visualizations confused users. The star-avatars represented the online community's users: the size represented the user's activity (upload/download), and the color represented the most common user's action (upload or download). The user with the biggest contribution was the sun in the sky, and the other users were randomly placed stars. The users wondered what the distance from the sun represented e.g., the closer to the sun, means closer to become the sun? Such meanings were excluded from the design.

In A3 the avatar-like images (i.e. smileys) were used as rewards after target achievement: all users had a sad smiley that became happy when they achieved their target. This helped the users see each other's target achievement.

To conclude, an anthropomorphized avatar that express emotions can engage users, which can be motivating. However, it is important to consider the negative effect of a sad avatar. A solution can be an avatar that only rewards a behavior (e.g., A3). Finally, using avatars in metaphorical environments (stars in the sky) should be thought carefully so that the metaphor conveys the intended meaning.

4.5.3. Graph like visualizations. These visualizations look like diagrams but lacking some of the diagram's characteristics e.g., well-formed $\mathrm{x}$ and $y$ axes, or because they include special characteristics, e.g., combination of different shapes.

A4 (and A6) visualized the users' data with a graph similar to a bar graph but without the axis, visualizing only integer values. The progress of the users was visualized with two bars: one for vegetable consumption and another for exercise. The bars consisted of small squares (slots) and each slot represented an action. For example, the first slot of the vegetable bar would be filled when the user had eaten one vegetable portion. The user could see the 
exact height of the bar because the bar itself showed how tall it is and how tall it could be.

On A9's public display, bars and lines were combined in the same circular diagram to represent electricity consumption. In A9 each user was represented by a bar that has as a starting point the circumference of a circle. The bars were grouped by color, each color represented a neighborhood. Moreover, two lines crossed the bars; one showing the average of the overall energy consumption, the other showing the neighborhood's average energy consumption.

The graph-like visualizations can be used to show a target or limit, e.g., you have to fill in 5 slots by eating 5 vegetable portions or you can eat only 5 sweets (e.g., each slot represents a sweet). Also, bargraph like visualizations can show the accumulative progress of all the users (e.g. A6). Finally, graph-like visualizations can be complicated but also more direct than avatars. For instance, it may be easier to understand if a user is above the average performance through a bar-like graph than by comparing different avatars scattered in their digital environment.

4.5.4. Traditional visualizations. This category includes any kind of traditional graphs and numeric representations of data (A5-A8, A10). A5 visualized the air quality of users' rooms with a bar and line graph. Similar graphs used by A7 to visualize the residences' water consumption. A8 used bar-graphs and colors to represent the ecofriendly actions the users took to commute to work. Each bar corresponded to a user and the different colors on it represented the transportation means used. The length of the bars represented the amount of "credits" given to users for choosing different transportation means.

One iteration of A6 used two numerical representations to visualize the fixture's average water consumption, and the current user's water consumption (counter-like). The users' feedback showed that numerical visualization made some users stressed, many related the numerical value to a specific goal, and it was less memorable and evoked less discussions between participants about the water consumption habits than the abstract visualization.

Two artifacts (A5, A7) examined the suitability of different visualizations for different people, and resulted that different people need different visualizations [8, 25]: families with children want visualizations that help their children learn, besides tracking their activities and comparing with others [8] young adults prefer the traditional graphs but seniors prefer aesthetics to fit their home decoration, if the artifact it is to be placed in the living room for example [25].
The traditional visualizations can show lots of information and give the possibility to manipulate data for creating different comparisons. However, complicated visualizations can be problematic for deploying public displays [4] and memorizing [17].

\subsection{Social comparison challenges}

The articles describe some challenges on designing IT artifacts for social comparison.

4.6.1. Fairness. In order for people to be motivated to change behavior, social comparison should be perceived as "fair". In two articles (A2, A7) the users wanted to be compared only with people similar to them. In A2 the users (students) rejected comparing with other disciplines' students. In A7 (target: water preservation) the users wanted to compare with households who had the same amount of inhabitants.

Social comparison theory [6] supports that the comparison should happen between people or group of people who have similar characteristics. For example, the comparison on water consumption between a house with garden and an apartment may demotivate the people living in the house and it may have been perceived unfair or pointless.

4.6.2. Consistency. Social comparison should be clear and influenced only by the compared values. For example, an iteration of A2 visualized only the users currently online in the community. Users knew that the star's size is influenced by their activity in the community. However, users who had big stars saw them shrinking, because others - who had made bigger contributions - logged in the system at the same time. Users found this confusing and difficult to comprehend. This may have a demotivating effect.

4.6.3. Data sharing. For having social comparison people's data need to be shared. None of the articles reported any problems on data sharing. Two articles related to A5 and A7 - examined users' consideration on air quality and water consumption data sharing. The users did not perceive these data as private.

Social comparison happens only if people share their data or at least their anonymized data. Thus, data related to health behavior change may be considered sensitive. However, no indication of this has been reported in the articles related to A1 and A3 regarding physical activity and eating related data.

4.6.4. Users' mental reaction. The comparison between the data of an individual and that of the others may stimulate unintentional competitions 
between users. For example [8], a family liked the idea of a virtual aquarium representing their water consumption status. However, they thought that visualizing the individual's effect, would stimulate unwanted competitive spirit instead of cooperative that it was promoted in their family.

How users perceive the comparison's attitude and users' personal philosophy should be in the same line. To avoid competitive attitude, a social comparison feature could show the users' data grouped e.g., in A6 the users compared their water consumption with the average water consumption.

\subsection{Summary}

In this paper, we created a design space for social comparison features - that visualize the data of an individual in relation to others - in technologies targeted to support behavior change.

One interesting aspect was that even though some of the articles we analyzed were based on the same theoretical model, they results in different

Table 2. Social comparaison design dimensions

\begin{tabular}{|c|c|}
\hline \multicolumn{2}{|c|}{ Strategies to influence behavior - How we want the artifact to support behavior change? } \\
\hline $\begin{array}{l}\text { Awareness } \\
\text { Immediate feedback }\end{array}$ & $\begin{array}{l}\text { Mainly promoted by public displays. } \\
\text { Promoted by technology directly connected to the activity. } \\
\text { Users have feed-back on their behavior at the place and time of action. } \\
\text { Make users aware of their behavior at the time of action. } \\
\text { Help users track their progress in comparison with others. } \\
\text { Usually, users have to input their own data. } \\
\text { The users have to actively enter the application to see their data. }\end{array}$ \\
\hline \multicolumn{2}{|c|}{ Types of comparisons - What comparison(s) will be represented? } \\
\hline $\begin{array}{l}\text { Compare with all users } \\
\text { Compare with aggregated users' data }\end{array}$ & $\begin{array}{l}\text { Users can see their position in the group in comparison with others. } \\
\text { Users can have a general understanding of their position in a group. } \\
\text { Users can have a general understanding of their impact on the group. } \\
\text { It may be perceived as less competitive. } \\
\text { Users can compare their data, with individuals and aggregated data. } \\
\text { More comparisons may lead to better perception of one's behavior. }\end{array}$ \\
\hline \multicolumn{2}{|c|}{ Dimensions of comparison - How many dimensions will the comparison have? } \\
\hline $\begin{array}{l}\text { One-dimensional } \\
\text { Two-dimensional } \\
\text { Multi-dimensional }\end{array}$ & $\begin{array}{l}\text { Shows the current relation between the users (suggested for ad-hoc feedback). } \\
\text { Shows the relation between the users in time/the users' progress. } \\
\text { Use visual cues (i.e. color, size) to show different comparison types. } \\
\text { Combination of visual cues' to represent many user's relations. }\end{array}$ \\
\hline \multicolumn{2}{|c|}{ Attitudes promoted - What design attitudes will be promoted? } \\
\hline Competition & $\begin{array}{l}\text { Usually, used together with gamified or metaphorical representations. } \\
\text { Rewarding users based on their individual progress. } \\
\text { Motivating them to be the best. } \\
\text { May demotivate users who dislike competition. } \\
\text { Promote team-spirit. } \\
\text { Can be combined with coopetition. } \\
\text { May promote accountability to the team-members. }\end{array}$ \\
\hline \multicolumn{2}{|c|}{ Visualizations of social comparison - How will we visualize social comparison? } \\
\hline Abstract visualizations & $\begin{array}{l}\text { Squares can show the user as part of a whole. } \\
\text { Color variation can show the relation between the users. } \\
\text { More memorable than traditional visualizations. } \\
\text { When in public they should draw attention, but be clear on their purpose. }\end{array}$ \\
\hline Avatars and avatar like visualizations & $\begin{array}{l}\text { Anthropomorphized or familiar form (i.e. star) visualizations. } \\
\text { They can visualize the state of the user. } \\
\text { They can be used as rewards. } \\
\text { Engage the users emotionally. }\end{array}$ \\
\hline Graph like visualizations & $\begin{array}{l}\text { Freedom of improvisation on shapes and colors. } \\
\text { Lack of axis ( } \mathrm{x} \text { and } \mathrm{y}) \text {. } \\
\text { Give a limit or target to the user. } \\
\text { Visualize more complicated data than avatar-like visualizations. }\end{array}$ \\
\hline Traditional visualizations & $\begin{array}{l}\text { Usually used to visualize a complex and detailed dataset of many users. } \\
\text { Allowed manipulation of the data and different comparisons. } \\
\text { Numerical visualizations may create a desire for a specific target/goal. } \\
\text { Preferred by young adults. } \\
\text { Not recommended for visualizations in public. }\end{array}$ \\
\hline \multicolumn{2}{|c|}{ Considerations on social comparison - What should we have in mind when designing for social comparison? } \\
\hline $\begin{array}{l}\text { Fairness } \\
\text { Consistency } \\
\text { Data sharing } \\
\text { Users' mental reaction }\end{array}$ & $\begin{array}{l}\text { Comparison only between users with similar characteristics. } \\
\text { Users' state should be influenced only by users' progress. } \\
\text { Privacy issues may be raised regarding anonymized and non-anonymized data. } \\
\text { The social comparison may be perceived as competition from users. } \\
\text { Different social influence features can result in different mental reactions. }\end{array}$ \\
\hline
\end{tabular}


designs. For example, Gasser et al. [10] and Lin et al. [19] refer to persuasive technology theory and the transtheoretical model, but their interpretation led them to different designs. Similarly, Sun and Vassileva [24] and Valkanova et al. [26] refer to social comparison theory but their artifacts resulted in different designs. Our results can act as a bridge between behavior change, social comparison, and persuasive technology theories/models on a more abstract level and the actual design of social comparison features on a practical level.

When comparing the dimensions that were implemented in health behavior artifacts (A1, A3, A5, A8) and artifacts in the context of ecological (A4, A6-A10) and other behavior (A2, A11), no clear difference could be identified. Even though "Compare with aggregated user's data", "Abstract visualizations", "Graph-like visualizations" and "Fairness" were only implemented in other than health-related artifacts, we cannot draw conclusions about the differences of social comparison features supporting health behavior and other behaviors due to the small number of artifacts. Our hypothesis is that many aspects are similar, but there may be some differences especially on data sharing.

Table 2 summarizes the six dimensions discussed above, the identified alternatives for each dimension, and design characteristics for each alternative. Additionally, six questions are presented that can be used to address the dimensions. Table 2 can be used by researchers as a basis to evaluate the different alternatives of each dimension; namely the suitability of the alternatives and their combinations in different contexts. Specifically, the questions can be used in design to reflect on design decisions or as a guide for the design process. Each dimension's alternatives can be used as examples or source of inspiration.

\section{Conclusions}

This paper presents a qualitative evidence synthesis review of 12 articles that describe studies of eleven IT artifacts targeting behavior change through visualization of the relationship between the data of an individual and that of others, among other social influence features. As a result, we present six social comparison design dimensions and alternatives for implementing them. Our results can be seen as a deeper examination of one social influence aspect (social comparison) and its implementation in technology that supports behavior change.

A limitation of the study is the small amount of articles examined. It is possible that more alternatives could have been uncovered for the dimensions, if we had a larger amount of artifacts to study. For the same reason, it was not possible to draw conclusions about the differences between health-related and other artifacts. Another limitation is our focus on social comparison using data of an individual and that of others. We did not study artifacts where, e.g., only groups were compared with other groups.

Our research has both theoretical and practical implications and opens up directions for future research. For researchers, our findings represent a repository of alternative solutions that allows for a systematic study and comparison of which design dimension and alternatives are best suited to achieve a desired behavior change in a specific context. For example, if we intend to implement healthier eating behavior, is there a difference in the outcome depending on whether the attitude promoted is competition or co-operation? Or are certain social comparison design dimensions are more influential in the context of health behavior change technology than in the context of ecological behavior? For practitioners, i.e., designers of behavior change technology, the six dimensions represent a concrete tool to lean on when deciding about the design/implementation of the technology. It can help them in getting inspiration, making design decisions, and being aware of their decisions' potential impacts.

\section{Acknowledgments}

This publication has received funding from the European Union's Horizon 2020 research and innovation programme - Marie Sklodowska-Curie Actions grant agreement no. 676201 - CHESS Connected Health Early Stage Researcher Support System.

\section{References}

[1] Aronson, E., The Social Animal, Macmillan, 2003.

[2] Beyer, H., and K. Holtzblatt., "Contextual Design" Interactions 6, 1999, pp. 32-42,

[3] Buunk, B. P, and F. X. Gibbons, Health, Coping, and Well-Being: Perspectives from Social Comparison Theory, Routledge, 2016.

[4] Chen, P. C., T. Jeng, Y. S. Deng, and S. F. Chien, "GreenSense: Developing Persuasive Service Technology by Integrating Mobile Devices and Social Interaction for Sustainable and Healthy Behavior", International Conference on Universal Access in Human-Computer Interaction, Springer, 2013, pp. 345-54.

[5] Collins, R. L., "For Better or Worse: The Impact of Upward Social Comparison on Self-Evaluations", Psychological Bulletin 119.1, 1996, pp. 51-69. 
[6] Festinger. L., "A Theory of Social Comparison Processes”, Human Relations 7.2, 1954, pp. 117-40.

[7] Fogg, B J., Persuasive Technology: Using Computers to Change What We Think and Do, Morgan Kaufmann, 2003.

[8] Froehlich, J., L. Findlater, M. Ostergren, S. Ramanathan, J. Peterson, I. Wragg, E. Larson, F. Fu, M. Bai, S. N. Patel, J. A. Landay, "The Design and Evaluation of Prototype Eco-Feedback Displays for Fixture-Level Water Usage Data", Proceedings of the SIGCHI Conference on Human Factors in Computing Systems, ACM, 2012, pp. 2367-76.

[9] Froehlich, J., L. Findlater, and J. Landay, "The Design of Eco-Feedback Technology", Proceedings of the SIGCHI Conference on Human Factors in Computing Systems, ACM 2010, pp. 1999-2008.

[10] Gasser, R., D. Brodbeck, M. Degen, J. Luthiger, R. Wyss, and S. Reichlin., "Persuasiveness of a Mobile Lifestyle Coaching Application Using Social Facilitation", Persuasive Technology, 2006, pp. 27-38.

[11] Gerrard, Meg, F. X. Gibbons, D. J. Lane, and M. L Stock, "Smoking Cessation: Social Comparison Level Predicts Success for Adult Smokers", Health psychology 24.6, 2005, pp. 623-29.

[12] Grant, M. J., and A. Booth,. "A Typology of Reviews: An Analysis of 14 Review Types and Associated Methodologies", Health Information \& Libraries Journal 26.2, 2009, pp. 91-108.

[13] Grevet, C, J. Mankoff, and S. D. Anderson, "Design and Evaluation of a Social Visualization Aimed at Encouraging Sustainable Behavior", Proceedings of the Annual Hawaii International Conference on System Sciences, IEEE, 2010, pp. 1-8.

[14] Guest G., K. M. MacQueen, E. E. Namey, Applied Thematic Analysis. SAGE, 2012.

[15] Gulbinas, R., R. K. Jain, and J. E. Taylor, "BizWatts: A Modular Socio-Technical Energy Management System for Empowering Commercial Building Occupants to Conserve Energy", Applied Energy 136, 2014, pp.1076-84.

[16] Gulbinas, R., and J. E. Taylor, "Effects of Real-Time Eco-Feedback and Organizational Network Dynamics on Energy Efficient Behavior in Commercial Buildings", Energy and Buildings 84, 2014, pp. 493-500.

[17] Kuznetsov, S., and E. Paulos, "UpStream: Motivating Water Conservation with Low-Cost Water Flow Sensing and Persuasive Displays", Proceedings of the 28th International Conference on Human Factors in Computing Systems, ACM, 2010, 1851-60.

[18] Langrial, S., A. Stibe, and H. Oinas-Kukkonen, "Practical Examples of Mobile and Social Apps Using the Outcome/change Design Matrix", PERSUASIVE (adjunct proceedings), 2013, pp. 7-13.

[19] Lin, J., L. Mamykina, S. Lindtner, G. Delajoux, and H.
Strub, "Fish'n'Steps: Encouraging Physical Activity with an Interactive Computer Game", UbiComp 2006: Ubiquitous Computing, 2006, pp. 261-78.

[20] Oduor, M., T. Alahäivälä, and H. Oinas-Kukkonen, "Persuasive Software Design Patterns for Social Influence", Personal and Ubiquitous Computing 18.7, 2014, pp. 1689-1704.

[21] Prochaska, J. O., and W. F. Velicer, "The Transtheoretical Change Model of Health Behavior", American Journal of Health Promotion 12.1, 1997, pp.3848.

[22] Stibe, A., "Towards a Framework for Socially Influencing Systems: Meta-Analysis of Four PLS-SEM Based Studies", International Conference on Persuasive Technology, Springer, 2015, pp. 172-183.

[23] Stibe, A, "Advancing Typology of ComputerSupported Influence: moderation Effects in Socially Influencing Systems", International Conference on Persuasive Technology, Springer, Cham, 2015, pp. 253-64.

[24] Sun, L., and J. Vassileva, "Social Visualization Encouraging Participation in Online Communities", Groupware: design implementation and use, 2006, pp.34963.

[25] Sunyoung, K., and E. Paulos, "InAir: Sharing Indoor Air Quality Measurements and Visualizations",

Proceedings of the SIGCHI Conference on Human Factors in Computing systems, ACM, 2010, pp. 1861-70.

[26] Valkanova, N, S. Jorda, M. Tomitsch, and A. V. Moere, "Reveal-It!: The Impact of a Social Visualization Projection on Public Awareness and Discourse", Proceedings of the SIGCHI Conference on Human Factors in Computing systems, ACM, 2013, pp. 3461-70.

[27] Valkanova, N., R. Walter, A. V. Moere, and J. Müller, "MyPosition: Sparking Civic Discourse by a Public Interactive Poll Visualization", Proceedings of the 17th ACM conference on Computer supported cooperative work \& social computing, ACM, 2014, pp. 1323-32.

[28] Wills, T. A., "Downward Comparison Principles in Social Psychology", Psychological Bulletin 90.2, 1981, pp. 245.

[29] Wohlin, C., "Guidelines for Snowballing in Systematic Literature Studies and a Replication in Software Engineering", 18th International Conference on Evaluation and Assessment in Software Engineering, ACM, 2014, pp.1-10.

[30] Wood, J. V, S. E. Taylor, and R. R. Lichtman, "Social Comparison in Adjustment to Breast Cancer", Journal of Personality and Social Psychology 49.5, 1985, pp.1169-83.

[31] Wunsch M., A. Stibe, A. Millonig, S. Seer, C. Dai, K. Schechtner, R. CC. Chin, "What Makes You Bike? Exploring Persuasive Strategies to Encourage Low-Energy Mobility", International Conference on Persuasive Technology, Springer, 2015, pp. 53-64. 